\section{Leitlinien für die Erbringung medizinischer Leistungen in der Diagnostik, Behandlung und Rehabilitation von Patienten mit Abhängigkeit und/oder schädlichem Gebrauch von psychotropen Substanzen für das Fachgebiet Psychiatrie/Psychotherapie}

\author{
(Stand 2000)
}

Ausgehend von den Auswirkungen der teilweise drastisch verkürzten Kostenzusagen für die stationäre Alkoholentzugsbehandlung auf die Behandlungsqualität und der potenziellen Ausgrenzung chronifizierungsgefährdeter Patienten haben sich im Frühjahr 1998 die leitenden Ärzte aller psychiatrischen Kliniken, welche für die regionale Pflichtversorgung Suchtkranker zuständig sind, im Bereich Nordrhein zusammengeschlossen. Aktiv einbezogen in diesen Diskussionsprozess wurden klinisch tätige Kollegen aus dem Bereich Westfalen-Lippe und niedergelassene Kollegen, vertreten durch den BVDN. Die gemeinsam von einer Redaktionsgruppe erarbeiteten und von allen Beteiligten verabschiedeten Leitlinien gehen über den ursprünglichen Anlass, die Sicherstellung adäquater Rahmenbedingungen für eine qualifizierte Alkoholentzugsbehandlung, deutlich hinaus. Mit der Formulierung der Leitlinien soll einer Spaltung zwischen Krankenhausbehandlung und medizinischer Rehabilitation, aber auch stationärer, teilstationärer und ambulanter Behandlung entgegengetreten und die gesamte Dimension der Behandlung suchtmittelmissbrauchender und suchtmittelabhängiger Menschen umfassend dargestellt werden. Der vollständige Text, der im Bereich Nordrhein bereits mit den Gesetzlichen Krankenkassen, der LVA und dem MDK diskutiert worden ist und auch der DHS, der DGPPN, VDR und BfA sowie der Ärztlichen Zentralstelle zur Zertifizierung vorliegt, ist bei den Autoren erhältlich; die Autorengruppe ist in der Legende abgedruckt.

\section{Präambel}

Der schädliche Gebrauch und/oder die Abhängigkeit von psychotropen Substanzen sind eigenständige krankhafte psychische Störungen. Sie haben in der Regel einen prozesshaften, langwierigen Verlauf und machen an sich und durch die substanzbedingten Folgeschäden die Erbringung medizinischer Leistungen im Sinne von Krankenbehandlung und/ oder medizinischer Rehabilitation notwendig. Die Abhängigkeitserkrankung ist den anderen psychischen Erkrankungen und Behinderungen (ICD 10, ICIDH, DSM IV) gleichgesetzt. Zur Erreichung therapeutischer Ziele ist immer eine sucht-

Suchttherapie 2001; 2: 45-50

(c) Georg Thieme Verlag Stuttgart · New York ISSN 1439-9903

\section{T. Marcea, Thomas Kuhlmann}

Arbeitsgemeinschaft „Sucht“ psychiatrischer Krankenhauseinrichtungen Nordrhein-Westfalen Arbeitskreis Sucht-Krankheiten des Berufsverbandes deutscher Nervenärzte, Landesverband Nordrhein spezifische psychiatrisch-psychotherapeutische Behandlung erforderlich.

In besonderen Fällen (z.B. im Vordergrund stehende somatische Folge- oder Begleiterkrankungen) sind die Leistungen anderer medizinischer Fachrichtungen (z. B. Innere, Chirurgie, Neurologie etc.) zeitweilig vorrangig. Rahmen, Inhalt und die Qualitätsmerkmale dieser Leistungen werden von den jeweiligen Fachverbänden definiert. Die erforderliche Integration dieser Behandlung in den individuellen suchtspezifischen psychiatrisch-psychotherapeutischen Therapieplan erfolgt durch enge Kooperation (z. B. Verlegung oder konsiliarische Zusammenarbeit) der somatisch behandelnden Stelle (Praxis, Krankenhaus) mit einer psychiatrisch-psychotherapeutischen Einrichtung bzw. einem psychiatrisch-psychotherapeutischen Facharzt.

Die für die Behandlung Abhängigkeitskranker notwendigen medizinischen Leistungen verstehen sich als Teile eines Behandlungs- und Hilfesystems. Dieses System umfasst neben den medizinischen Leistungen u.a.: Suchtberatung, berufliche Rehabilitation, soziale Leistungen der Eingliederungshilfe, Selbsthilfe-Organisationen, sozialpsychiatrische Dienste, komplementäre Einrichtungen.

Das Behandlungs- und Hilfsangebot für Abhängigkeitskranke ist sehr komplex. Konsensfähige Kriterien für den Zugang, den Inhalt und Umfang der Leistungen fehlen weitgehend. Dadurch wurde die Erbringung medizinischer Leistungen bei Abhängigkeit und/oder schädlichem Gebrauch von psychotropen Substanzen zunehmend problematisch und eine effiziente Vernetzung im Sinne der Abhängigkeitskranken erschwert. Es erschien uns deswegen notwendig, eine Arbeitsgemeinschaft zu gründen mit der Aufgabe, durch Konsens von Experten diesbezüglich orientierende Leitlinien $\mathrm{zu}$ formulieren.

Das Hauptanliegen dieser Leitlinien ist, den Beteiligten (Abhängigkeitserkrankte, Leistungserbringer, Kosten- und Leistungsträger) die für die Behandlung der Abhängigkeitserkrankung notwendigen medizinischen Leistungen und deren Grundlage nachvollziehbarer zu machen und somit Entscheidungshilfen zu geben. 
Diese Leitlinien sind durch Konsensfindung entstanden, sie vertreten den fachlichen Standpunkt der Erbringer medizinischer Leistungen unter Berücksichtigung des Wissensstandes aufgrund wissenschaftlicher Untersuchungen und empirischer Erkenntnisse sowie der gesetzlichen Bestimmungen.

Die Arbeitsgemeinschaft hat eigenständig gearbeitet und hat bei der Leitlinienentwicklung keinerlei Unterstützung durch Dritte erhalten.

Es ist vorgesehen, diese Leitlinien spätestens alle 5 Jahre oder bei grundlegenden Veränderungen des diesbezüglichen Wissenstandes und/oder der gesetzlichen Grundlage $\mathrm{zu}$ revidieren.

\section{Abschnitt 1}

Grundlegende Aspekte der Erbringung medizinischer Leistungen bei schädlichem Gebrauch oder Abhängigkeit von psychotropen Substanzen

\subsection{Anspruch auf medizinische Leistungen}

Anspruch auf medizinische Leistung bei Missbrauch von psychotropen Substanzen besteht zum diagnostischen Zwecke und wenn nach ICD 10 oder DSM IV bzw. ICIDH ein schädlicher Gebrauch/eine Abhängigkeit von psychotropen Substanzen diagnostiziert und/oder in Zusammenhang damit entstandene weitere Krankheiten bzw. Behinderungen ärztlich festgestellt wurde.

\subsection{Gesetzliche Grundlage für die Kosten- und Leistungsträgerschaft}

Die Erbringung medizinischer Leistungen wegen gesundheitlicher Störungen bei schädlichem Gebrauch/Abhängigkeit von psychotropen Substanzen werden, wie bei anderen Erkrankungen, von SGB V und VI sowie BSHG wie folgt geregelt:

\subsubsection{Medizinische Leistungen zur Krankenbehandlung}

Laut §27 SGB V haben Versicherte „Anspruch auf Krankenbehandlung, wenn sie notwendig ist, um eine Krankheit zu erkennen, $\mathrm{zu}$ heilen, ihre Verschlimmerung zu verhüten oder Krankheitsbeschwerden zu lindern“. Nichtversicherte haben den gleichen Anspruch wie die Versicherten (BSHG §§36, 37 ff.). Leistungen der Krankenbehandlung bei schädlichem Gebrauch/Abhängigkeit von psychotropen Substanzen sind die Diagnostik und die Behandlung: Detoxikation, Behandlung der Folge- und/oder Begleiterkrankungen, Förderung der Krankheitsbewusstwerdung (Einsicht), Erarbeitung von Strategien zur Überwindung bzw. Bewältigung der Krankheit im Rahmen eines Behandlungsplans.

\subsubsection{Medizinische Leistungen zur Rehabilitation}

Es handelt sich um medizinische Behandlung mit den Zielen, die Krankheitsfolgen $\mathrm{zu}$ beheben oder $\mathrm{zu}$ mindern, noch vorhandene Fähigkeiten zu mobilisieren, um ggf. die verbliebene Behinderung zu kompensieren, sowie den Auswirkungen dieser Behinderung „auf die Erwerbstätigkeit entgegenzuwirken oder sie zu überwinden“ ( $§ 9$ (1) sowie $\S \S 10$,
15 SGB VI). Voraussetzungen für die Gewährung von medizinischen Leistungen der Rehabilitation: Rehabilitationsbedürftigkeit, Rehabilitationsfähigkeit, Erfolgsaussicht. Eine zentrale Zielsetzung der ärztlichen Leistung in der Rehabilitation Abhängigkeitskranker ist die Loslösung des Betroffenen aus der Abhängigkeit von seiner Suchtsubstanz.

Die Kosten der Rehabilitation werden gemäß SGB VI von den Rentenversicherungen getragen.

Subsidiär können medizinische Leistungen zur Rehabilitation auch auf Grundlage von SGB V $(\S \S 11,27,40,43)$ finanziert werden. Nichtversicherte haben ebenfalls Anspruch auf Leistungen zur Rehabilitation (BSHG $\S \S 37,43$ ).

\subsection{Grundsätzliche Aspekte in Zielsetzung und Inhalt der medizinischen Leistungen bei schädlichem Gebrauch/ Abhängigkeit von psychotropen Substanzen}

Grundsätzlich soll jede Behandlungsstrategie die langfristige Befreiung des Patienten aus der Abhängigkeit von psychotropen Substanzen und die Überwindung der gegebenenfalls entstandenen Gesundheits- und sozialen Schäden zum Ziel haben.

Wenn diese Behandlungsziele nicht sofort oder mittelfristig erreicht werden können, soll das Erreichen von Teilzielen (z. B. zeitweilige Abstinenz) als wichtige Bausteine des Behandlungs- und Gesundungsverlaufs angestrebt werden.

Neben der psychiatrisch-psychotherapeutischen sowie rehabilitativen Behandlung können auch pharmakotherapeutische Mittel (z.B. Antagonisten, Aversiva, Anticraving-Substanzen) eingesetzt werden.

In besonderen Fällen (z.B. schwere körperliche Erkrankung, Schwangerschaft bei Drogenabhängigkeit vom Opiat-Typ) kann die Therapie vorübergehend oder langfristig eine kontrollierte Substitution der Suchtsubstanz mit einem ärztlich verordneten Agonisten (z.B. Methadon) sein, gemäß geltenden Richtlinien der Bundes- und Landesärztekammern.

\subsubsection{Medizinische Leistungen der Krankenbehandlung}

Diagnostik: Feststellung, ob ein schädlicher Gebrauch/eine Abhängigkeit von psychotropen Substanzen vorliegt und welche Gesundheitsstörungen entstanden sind:

- Körperliche Diagnostik: akute und chronische Folgen der Substanzeinwirkung auf den Körper; Folgen der Detoxikation (Entgiftung); indirekte Folgen des Substanzmissbrauchs (z.B. durch Beeinträchtigung der Lebensweise, Ernährung etc.).

- Psychiatrische Diagnostik: Erkennen des substanzabhängigen Verhaltens, psychischer Folgen der Detoxikation, des schädlichen Gebrauchs, ggf. einer psychiatrischen Komorbidität.

- Soziale Diagnostik: vorhandene und drohende Auswirkung und Interaktionen des schädlichen Gebrauchs/Abhängigkeit von psychotropen Substanzen im sozialen Umfeld, insbesondere auf die Erwerbsfähigkeit des Betroffenen.

Therapie: Der schädliche Gebrauch/die Abhängigkeit von psychotropen Substanzen ist in der Regel eine langwierig verlaufende Erkrankung und bedarf deshalb einer ebenfalls 
auf eine lange zeitliche Perspektive angelegten therapeutischen Strategie im Sinne eines Behandlungsplans. Die nachfolgend angegebenen Aspekte der Therapie sind deshalb als Teile eines Ganzen zu verstehen. Sie sind in jedem Behandlungsfall und in jeder Behandlungsphase in unterschiedlicher Ausprägung zu berücksichtigen. Die medizinischen Leistungen der Krankenbehandlung haben als Ziel, den schädlichen Gebrauch/die Abhängigkeit von psychotropen Substanzen sowie die gegebenenfalls begleitenden weiteren Gesundheitsschäden spezifisch zu behandeln.

- Detoxikation: Behandlung der psychischen und organischen Folgen der Intoxikation und Eliminierung der psychotropen Substanz, Hilfe bei der Überwindung der Entzugserscheinungen.

- Behandlung der etwaigen dem schädlichen Gebrauch/der Abhängigkeit von psychotropen Substanzen zugrunde liegenden Erkrankungen sowie der psychischen und/oder organischen Folge- und/oder Begleiterkrankungen.

- Förderung der Krankheitsbewusstwerdung (Einsicht), Aufbau eines individuellen Krankheitsmodells als Grundlage für den Behandlungsplan.

- Erarbeitung von Strategien zur Krankheitsüberwindung bzw. Bewältigung oder Begrenzung der Folgen, Motivieren und Befähigen zur Umsetzung dieser Strategien (u.a. Stärkung zur Eigenverantwortung und der Realitätsprüfung) unter Berücksichtigung der individuellen Verhältnisse.

\subsubsection{Medizinische Leistungen zur Rehabilitation}

Diagnostik: Vervollständigung der Diagnostik um die für die Rehabilitationsmaßnahmen notwendigen Erkenntnisse:

- Körperliche Diagnostik: akute und chronische, reversible und irreversible Folgen der Substanzeinwirkung auf den Körper; indirekte Folgen des Substanzmissbrauchs (z.B. Ernährungsstörungen, körperliche Schäden, Begleiterkrankungen etc.); weitere, vom schädlichen Gebrauch von psychotropen Substanzen unabhängige Krankheiten.

- Psychische Diagnostik: Überprüfung der Indikation und vertiefte Analyse des substanzabhängigen Verhaltens; psychische Folgen der Detoxikation; akute, chronische, reversible und irreversible psychische Folgen des Substanzmissbrauchs; evtl. Begleiterkrankungen und sozialmedizinisch relevante Aspekte.

- Soziale Diagnostik: Auswirkungen und Interaktionen des schädlichen Gebrauchs/Abhängigkeit von psychotropen Substanzen im sozialen Umfeld, besonders auf die Erwerbsfähigkeit des Betroffenen, Ermittlung des Ausmaßes akuter, chronisch vorhandener und drohender Störungen.

Therapie: Behandlung der im Zusammenhang mit dem schädlichen Gebrauch/der Abhängigkeit von psychotropen Substanzen entstandenen psychischen, organischen und sozialen Störungen und der zugrunde liegenden psychischen und/oder organischen und sozialen Störungen mit den Zielen:

- die Erwerbstätigkeit wesentlich zu bessern oder wiederherzustellen und den Eintritt der Berufs- oder Erwerbsunfähigkeit abzuwenden.

- bei Nichterwerbstätigen bezwecken die Rehabilitationsmaßnahmen die Wiederherstellung oder Besserung somatischer und psychosozialer Fähigkeiten, sie sollen das mögliche Eintreten der Pflegebedürftigkeit abwenden.
Um diese Ziele zu erreichen, werden in der Rehabilitation folgende medizinische Leistungen erbracht:

- Behandlung von etwaigen dem schädlichen Gebrauch/der Abhängigkeit von psychotropen Substanzen zugrunde liegenden Erkrankungen, der Folge- und/oder Begleiterkrankungen (psychische und/oder organische), sofern zur Erreichung der Rehabilitationsziele erforderlich und mit den Mitteln der Rehabilitationsmaßnahmen erreichbar.

- Weiterentwicklung und Vertiefung des individuellen Krankheitsmodells, therapeutisches Bündnis, Aufarbeitung der Auswirkungen der mit dem schädlichen Gebrauch/der Abhängigkeit von psychotropen Substanzen einhergehenden Störungen auf alle Lebensbereiche (biopsychosoziales Modell).

- Erarbeitung von Strategien zur Krankheitsüberwindung bei Begrenzung der Folgen. Motivieren und Befähigen zur Umsetzung dieser Strategien (u.a. Eigenverantwortung, Realitätsprüfung) auf allgemeinmedizinischer, psychotherapeutischer und handlungsorientierter Ebene.

\section{Mitarbeit der Betroffenen}

Die medizinische Leistung zur Krankenbehandlung sollte in der Regel auf Wunsch und unter Mitarbeit der Betroffenen erbracht, kann aber in besonderen Fällen auch durch Zwangsmaßnahmen ermöglicht werden.

Bei medizinischen Leistungen in der Rehabilitation sind die Betroffenen zur Mitarbeit verpflichtet (§9 (2) SGB VI).

Die Motivation des Betroffenen zur Mitarbeit ist ein dynamischer Prozess und soll in der aktuellen Situation immer neu überprüft werden. Die zeitweilige Unfähigkeit einer Patientin oder eines Patienten zur kontinuierlichen Mitarbeit ist als Symptom der Suchterkrankung oder der der Suchterkrankung zugrunde liegenden psychischen Störung zu verstehen. Aus der vorübergehenden fehlenden Mitarbeit des Betroffenen dürfen diesem daher keine Nachteile erwachsen.

\section{Abschnitt 2}

\section{Auswahl der Leistungsart}

Gemäß SGB V (§§2 (4), 12 (1)) müssen medizinische Leistungen der Krankenbehandlung im Allgemeinen folgende Kriterien erfüllen: Sie sollen notwendig, zweckmäßig, wirksam, ausreichend und wirtschaftlich sein. In der Qualität und Wirksamkeit haben diese Leistungen dem allgemeinen Stand der medizinischen Erkenntnisse zu entsprechen und den medizinischen Fortschritt zu berücksichtigen. Ambulante Behandlung ist gegenüber der teil- bzw. vollstationären Behandlung vorrangig.

Unter Berücksichtigung dieser gesetzlichen Grundlagen werden die notwendigen medizinischen Leistungen zur Krankenbehandlung, in der individuell geeigneten Form, bis zur Erreichung therapeutischer Ziele gewährt.

Die Behandlungsindikation wird vom Arzt festgelegt. Die Notwendigkeit der Krankenhausbehandlung wird nach §39 (1) SGB V vor der Aufnahme durch das Krankenhaus geprüft. Die BSHG-Regelung ist analog. 


\subsection{Institutionelle Formen der medizinischen Leistungen bei schädlichem Gebrauch/Abhängigkeit von psychotropen Substanzen}

\subsubsection{Medizinische Leistungen der Krankenbehandlung}

Grundsätzlich sind alle Ziele der medizinischen Leistungen zur Krankenbehandlung mit jeder einzelnen Form der institutionalisierten Behandlung alleine erreichbar.

Eine vollstationäre Krankenhausbehandlung ist laut SGB V, $\S 39$ (1) nur dann zu erbringen, wenn das therapeutische Ziel nicht durch ambulante oder teilstationäre Behandlung erreicht werden kann.

Ambulante Behandlung ist ausreichend, wenn die im Kapitel 1.3 ausgeführten Ziele mit dieser Behandlungsform erreichbar sind und die Ausprägung der psychischen und körperlichen Symptome keine ambulant nicht beherrschbaren Komplikationen erwarten lässt (z.B. in der Entgiftungsphase), der Betroffene zuverlässig mitarbeitet und das soziale Umfeld (z.B. Familie) ausreichend unterstützend ist.

Die ambulante Behandlung ist nicht ausreichend, wenn diese Voraussetzungen nicht erfüllt sind.

Die Erbringung ambulanter medizinischer Leistungen zur Krankenbehandlung erfolgt im Rahmen des Sicherstellungsauftrages (§72 SGBV) durch niedergelassene Ärzte und Psychotherapeuten (gemäß Psychotherapie-Richtlinien), durch Institutsambulanzen sowie vor- und nachstationär (§§39.1., 115 a, 118 SGBV).

Krankenhausbehandlung (vollstationär und teilstationär) ist notwendig, wenn die im Kapitel 1.3 ausgeführten Ziele nicht ambulant zu erreichen sind und/oder die ambulante Behandlung von medizinisch nicht vertretbaren Risiken behaftet ist (z.B. unberechenbare oder schwere körperliche Entzugserscheinungen, körperliche Begleiterkrankungen, Suchtfolgeerkrankungen, Psychose, Suizidalität).

Krankenhausbehandlung (teil- oder vollstationär) ist des Weiteren notwendig, wenn für die Erreichung therapeutischer Ziele die zeitweilige Herausnahme des Patienten aus dem bisherigen System und/oder die spezifische Wirkung der psychiatrisch-psychotherapeutischen Krankenhausstrukturen erforderlich sind.

Die Behandlung wird teilstationär durchgeführt, wenn das Erreichen der Behandlungsziele auch bei verminderter Betreuungs- und Überwachungszeit sowie bei gegenüber der vollstationären Behandlung reduzierten therapeutischen Angeboten voraussichtlich möglich ist. Ist eine teilstationäre Behandlung möglich, so ist diese der vollstationären vorzuziehen, wenn eine verstärkte Auseinandersetzung mit der Alltagsrealität gefordert werden muss, mit der therapeutischen Zielsetzung, die dadurch entstandenen Probleme zeitnah zu bearbeiten.

Die vollstationäre Behandlung ist der teilstationären Behandlung immer dann vorzuziehen, wenn die Krankheitsrisiken und/oder die Erfordernisse für die Erreichung der therapeutischen Ziele die ständige Wirkung der psy- chiatrisch-psychotherapeutischen Krankenhausstrukturen, insbesondere die ständige ärztliche Präsenz notwendig machen.

\subsubsection{Medizinische Leistungen zur Rehabilitation}

Die gemäß SGB V, §40 und SGB VI, §§9 $(1), 15(1,2)$ gewährten medizinischen Leistungen zur Rehabilitation können je nach Erfordernis ambulant oder stationär durchgeführt werden (SGB VI §13 (1)).

Ambulante medizinische Rehabilitation ist ausreichend, wenn die im Kapitel 1.3.2 ausgeführten Ziele mit dieser Behandlungsform erreichbar sind und aufgrund der Ausprägung der psychischen und körperlichen Symptome der bisherige Krankheitsverlauf sowie die soziale Situation des Betroffenen eine stationäre Leistung zur medizinischen Rehabilitation aktuell nicht erforderlich machen. Eine ambulante medizinische Rehabilitation ist auch dann angezeigt, wenn Teilnehmer an einer stationären Rehabilitation bei noch nicht abgeschlossener Entwöhnungsbehandlung so weit fortgeschritten sind, dass die Maßnahme nicht mehr stationär erfolgen muss. Die ambulante Rehabilitation kommt nicht in Betracht, wenn diese Voraussetzungen nicht erfüllt sind.

Eine stationäre (vollstationäre und/oder teilstationäre) medizinische Rehabilitation ist notwendig, wenn die im Kapitel 1.3.2 ausgeführten Ziele nicht ambulant zu erreichen sind und/oder die ambulante Rehabilitation von medizinisch oder sozial nicht vertretbaren Risiken behaftet ist (z.B. Ausmaß der körperlichen und psychischen Erkrankungen, Entfernung der Therapieeinrichtung vom Wohnort, häusliche Situation etc.).

Eine stationäre medizinische Rehabilitation ist des Weiteren notwendig, wenn für die Erreichung therapeutischer Ziele die zeitweilige Herausnahme des Patienten aus dem bisherigen System und/oder die spezifische Wirkung der stationären Strukturen der Einrichtung zur medizinischen Rehabilitation erforderlich sind.

\subsection{Formen der medizinischen Leistungen bei schädlichem Gebrauch/Abhängigkeit von psychotropen Substanzen nach therapeutischen Schwerpunkten und Zeitrahmen}

Die nachfolgend ausgeführten medizinischen Leistungen können gemäß ärztlicher Indikation ambulant, teilstationär oder stationär erbracht werden.

\subsubsection{Medizinische Leistungen der Krankenbehandlung}

Im Mittelpunkt der Behandlung stehen suchtspezifische psychiatrische und psychotherapeutische Leistungen, welche unmittelbar in Zusammenhang mit dem Substanzmissbrauch/der Abhängigkeit notwendig geworden sind.

Es gibt in diesem Rahmen 3 Behandlungssettings, die Teile einer Gesamtstrategie sein können. Die Dauer der Behandlung hängt von der Ausprägung der Krankheit und der Erreichung der daraus notwendig gewordenen therapeutischen Ziele ab.

(Vermerk: Bei der Darstellung nachfolgender Behandlungssettings wurde auf missverständliche Begriffe „Entgiftung“ und „Entzug“ verzichtet). 
Kurzintervention: Es handelt sich um eine kurzzeitige, suchtspezifische psychiatrisch-psychotherapeutische Behandlung mit den therapeutischen Schwerpunkten Detoxikation und Krisenintervention. Die Dauer der Behandlung richtet sich nach der Ausprägung der durch den Substanzmissbrauch/die Abhängigkeit verursachten Symptome und Begleiterkrankungen.

Indikationen:

- diese Behandlung ist aufgrund der Krankheitsvorgeschichte und der aktuellen Zielsetzung derzeit ausreichend,

- der Patient lehnt eine weitere Behandlung ab.

Diese Behandlungsform kann Eingangsstufe für eine vertiefte Therapie sein.

Vertiefte suchtspezifische psychiatrische psychotherapeutische Behandlung. Die grundsätzlichen Schwerpunkte dieser Behandlung sind, neben der somatischen Therapie: Bewusstwerdung der eigenen Abhängigkeitserkrankung, Aufbau eines individuellen Krankheits- und Gesundheitskonzeptes sowie die Erarbeitung von längerfristigen Behandlungsstrategien.

Indikationen:

- Die Behandlungsmotivation ist eingeschränkt bzw. labil, sie kann aber voraussichtlich gefestigt werden.

- Eine Änderungsmotivation lässt sich voraussichtlich weiterentwickeln oder konkretisieren.

Behandlung mehrfach erkrankter Abhängiger: Es handelt sich hier in der Regel um schwere, komplexe, langdauernde, in Verbindung mit Substanzmissbrauch/Abhängigkeit entstandene psychiatrische Erkrankungen sowie psychiatrische Grund- und Begleiterkrankungen, welche längerfristiger, ineinander greifender und vernetzter Behandlungsstrategien bedürfen (z.B. hirnorganische psychische Störungen, Psychosen, Anpassungsstörungen, Persönlichkeitsstörungen etc.).

\subsubsection{Medizinische Leistungen der Rehabilitation („Entwöhnungsbehandlung“)}

Kurzzeit-Rehabilitationsbehandlung: mehrwöchige Therapie mit dem Ziel der fokussierten Bearbeitung spezifischer Problem- und Konfliktfelder und der zielgerichteten ressourcenorientierten Stabilisierung zur Bewältigung und Überwindung der Abhängigkeitsproblematik.

Indikation:

- stabile Behandlungsmotivation und Krankheitseinsicht mit guter Einbindung in vor- und nachstationäre Hilfsangebote.

- u.U. nach vorheriger LZ-Therapie mit nur kurzfristigem Rückfall ohne umfassende psychische, physische und/oder psychosoziale Dekompensation.

Langzeit-Rehabilitationsbehandlung: mehrmonatige Therapie mit dem Ziel der umfassenden Bearbeitung der Entwicklung und aktuellen Ausprägung der Abhängigkeitsproblematik und der ressourcenorientierten Erarbeitung von Strategien zur längerfristigen Stabilisierung.

\section{Indikation:}

- vorhandene, u.U. noch labile Behandlungsmotivation und Krankheitseinsicht bei ausgeprägten psychischen, somatischen und/oder psychosozialen Problemen
- gegebene Einbindung in das psychosoziale Suchthilfeangebot ist wünschenswert, jedoch nicht zwingend erforderlich.

\section{Redaktionsgruppe}

I. T. Marcea, Dr., Ärztlicher Direktor der Psychiatrischen Klinik, Marienborn, 53909 Zülpich

Thomas Kuhlmann, Dr., Chefarzt der Psychosomatischen Klinik, Schlodderdicher Weg 23a, 51469 Bergisch Gladbach

P. Summa-Lehmann, Dr., Chefarzt der Suchtabteilung, Rheinische Kliniken, 52353 Düren

Gerhard Reymann, Dr., Leitender Abteilungsarzt Suchtmedizin am Westfälischen Zentrum für Psychiatrie, Psychotherapie und Psychosomatik, 44287 Dortmund

Th. Reinert, Dr., Chefarzt, Fachklinik Langenberg, 42555 Velbert-Langenberg

J. Endres Dr., Abteilungsarzt (Bereich Sucht) des St. AlexiusKrankenhauses - Fachkrankenhaus für Psychiatrie und Neurologie, 41403 Neuss

M. Lütz, Dr., Chefarzt, Alexianer-Krankenhaus, 51149 KölnPorz

A. Gilles, Dr., Leitende Ärztin, Suchtklinik - Alexianer-Krankenhaus, 47805 Krefeld

H. Elsner, Dr., Oberarzt, Abteilung für Psychiatrie und Psychotherapie, Elisabeth-Krankenhaus GmbH, 45891 Gelsenkirchen

J. Plath, Chefarzt Rheinische Kliniken Köln, Suchtabteilung, 51076 Köln

R. Peters, Dr., BVDN - Landesverband Nordrhein -, Sektion Psychiatrie, Psychotherapie, BVDP, Referat Sucht, 52428 Jülich

H. Beuthner, Dr., BVDN - Landesverband Nordrhein -, Arbeitskreis Sucht, 47198 Duisburg

S. Schreckling, Dr., BVDN, Landesverband Nordrhein, Sektion Psychiatrie, Arbeitskreis Sozialpsychiatrie, 50354 Hürth

\section{Unter Mitwirkung von:}

Priv. Doz. Dr. M. Banger (Essen), P. Bommersbach (Euskirchen), A. Brunk (Duisburg-Walsum), J. Eufinger (Duisburg), Dr. W. Gerlich (Bonn), Prof. Dr. Dr. M. Hambrecht (Köln), Dr. P. Hunoldt (Bonn), Dr. G. Kotschick (Euskirchen), Prof. Dr. Kröger (Ratingen), Prof. Dr. F. Matakas (Köln), E. Max (Viersen), Dr. H. Pelzer (Duisburg), Dr. D. Petzsche (Langenfeld), Dr. A. Schacht (Gangelt), Dr. W. Schroeder (Ratingen), Dr. M. Seel-Wicke (Viersen), B. Voss (Köln), Dr. Hermann Westendarp (BrilonWald), T. Wiese (Duisburg), Prof. Dr. K. Windgassen (Remscheid), D. Zieger (Aachen) 


\section{Literatur}

${ }^{1}$ Aktion Psychisch Kranke. Innovative Behandlungsstrategien bei Alkoholproblemen. Lambertus, 1997

${ }^{2}$ APA (American Psychiatric Association). Substance Use Disorders - Alcohol, Cocaine, Opioids. In Practice Guidelines. APA Washington. D.C, 1996

${ }^{3}$ Bundesarbeitsgemeinschaft für Rehabilitation. Arbeitshilfe für die Rehabilitation von Suchtkranken - Alkohol - Drogen Medikamente, 1996; 12

${ }^{4}$ Fachverband Sucht e.V. Gesamtkonzept des Fachverbandes Sucht e.V. zur Behandlung von Abhängigkeitskranken. Reihe: Qualitätsförderung in der Entwöhnungsbehandlung, 1998; 5

${ }^{5}$ Ministerium für Arbeit, Gesundheit und Soziales, Nordrhein Westfalen (Referat VAZ. AZ 039258). Anmerkungen und Hinweise zur Erstellung von Einzelkonzepten für eine qualifizierte Drogenentzugsbehandlung

${ }^{6}$ DSM IV. In: Saß H, Wittchen HV, Zandig M (Hrsg). Hogrefe Verlag für Psychologie, 1996

${ }^{7}$ ICD 10, Kap. V (F). In: Dilling H, Mambour W, Schmidt MH (Hrsg). Verlag Hans Huber, 1993

${ }^{8}$ ICIDH - Internationale Klassifikation der Schädigungen, Fähigkeitsstörungen und Beeinträchtigungen. In: Matthesius RG, Jochheim KA, Barolin GS, Heinz C (Hrsg)., 1995

${ }^{9}$ BSHG Walhalla Fachverlag, (Stand 1999)

${ }^{10}$ SGB. Essen Fachverlag CW Haarfeld GmbH, 1998

${ }^{11}$ Rheinische Arbeitsgemeinschaft für Rehabilitation (Rundschreiben vom 6.6.1979). Stationäre Entwöhnungsbehandlung, Empfehlungsvereinbarung über die Zusammenarbeit der KV und RV bei der Rehabilitation Abhängigkeitskranker vom 20.11.1978. Düsseldorf

\section{Dr. I. T. Marcea}

Ärztlicher Direktor der Psychiatrischen Klinik Marienborn 53909 Zülpich

\section{Dr. Thomas Kuhlmann}

Chefarzt der Psychosomatischen Klinik Schlodderdicher Weg 23a

51469 Bergisch Gladbach 